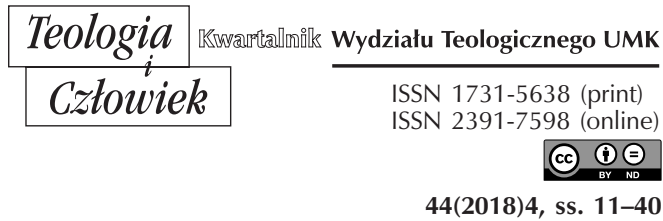

KS. RYSZARD SELEJDAK

\title{
LE DISPOSIZIONI RIGUARDANTI IL DIACONATO PERMANENTE DEI CONCILI DAL IV AL XVI SECOLO
}

DOI: http://dx.doi.org/10.12775/TiCz.2018.037

Streszczenie. Posługa diakonatu stałego przedstawiona jest już w niektórych tekstach Nowego Testamentu. Była ona niezwykle ważna dla życia wspólnot chrześcijańskich, zwłaszcza w Kościele starożytnym. W średniowieczu przeżywała głęboki kryzys. Wtedy to diakonat zaniknął jako stała posługa, stając się jedynie „krokiem”, „stopniem” prowadzącym do kapłaństwa, połączonym z liturgicznymi i administracyjnymi zadaniami pełnionymi przez diakonów z polecenia biskupa. Sobory odbywające się w Kościele zarówno na Zachodzie, jak i na Wschodzie od IV do XVI wieku wydały szereg dyspozycji dotyczących statusu osobistego i funkcjonalnego diakonów. Soborowe dekrety i kanony dotyczyły w szczególności tożsamości prawnej i teologicznej diakonów, ich sakramentalnych prerogatyw, wykonywanych zajęć, funkcji liturgicznych, celibatu, dolnej granicy wieku dopuszczenia do święceń, sposobu ubierania się i zachowania. Szczególną uwagę poświęciły działalności charytatywnej diakonów jako najbardziej ich charakterystycznej posługi. Postanowienia soborowe były często reakcją na nadużycia, które zdarzały się w życiu Kościoła.

Słowa kluczowe: sobór; sakrament święceń; diakon stały; liturgia.

Abstract. A Conciliar View of the Permanent Diaconate Between the $4^{\text {th }}$ and $16^{\text {th }}$ Century. The ministry of the permanent diaconate appears in several New Testament texts. At the dawn of Christianity, it played a significant role in the service of 
the Christian community. In Medieval times, it suffered a steady decline that led to its eventual disappearance as a permanent ministry, becoming solely a "step" on the path to priesthood, thus associated with the liturgical and administrative task of the Bishop. The Church Councils, in the West as in the East, from the $4^{\text {th }}$ to $16^{\text {th }}$ cent., issued various teachings on the personal and functionary status of deacons. Conciliar decrees and canons focused on the juridical and theological identity of the diaconate especially, its sacramental character, tasks to be performed, liturgical functions to be administered, celibacy, the minimum age of ordination, the dress code and conduct. Much emphasis was placed upon the charitable activity of deacons, understood as pertaining primarily to them. The Conciliar positions on the diaconate were oftentimes a response to some form of abuse in the life of the Church.

Key words: Council; Holy Orders; Permanent Deacon; Liturgy.

\section{INTRODUZIONE}

Per attuare l'aggiornamento della Chiesa, il Concilio Vaticano II ha cercato, nelle sue origini e nella sua storia, ispirazione e mezzi per annunciare e rendere presente in maniera più efficace il mistero di Gesù Cristo. Tra queste ricchezze della Chiesa si trova il ministero del diaconato, del quale i testi del Nuovo Testamento rendono testimonianza. Tale ministero ha reso importanti servizi alla vita delle comunità cristiane soprattutto ai tempi della Chiesa primitiva. Entrato in declino nel Medioevo, esso è scomparso come ministero permanente, sussistendo solamente come transizione verso il presbiterato e l'episcopato. Ciò non ha impedito che dal tempo della scolastica sino ai nostri giorni ci si interesasse del suo significato teologico e, in particolare, del problema del suo valore sacramentale come grado dell'ordine. Il presente studio vuole presentare le disposizioni più importanti dei Concili svoltosi dal IV al XVI secolo sullo statuto personale e sullo statuto funzionale dei diaconi permanenti nelle comunità cristiane.

\section{CONCILI ANTICHI}

Un Concilio particolarmente interessante per la suddetta problematica fu quello di Elvira. Tale Concilio si svolse in Spagna intorno al 300 e 
costituì un importante riferimento per le normative disciplinari emanate ${ }^{1}$.

Tali disposizioni, relativamente allo statuto diaconale, imponevano primariamente sul piano personale il celibato:

Si è stabilito di proibire assolutamente ai vescovi, presbiteri e diaconi e a tutti i membri del clero posti nel ministero, ogni coniugio, e di generare figli; chi lo farà, sia escluso dal clero² .

Si tratta della prima disposizione conosciuta che imponga il celibato non solo a coloro che hanno ricevuto lordine ma anche agli altri ministeri. A questa norma erano collegate anche quelle di natura più prudenziale:

Il Vescovo e ogni altro chierico abbia con sé o una sorella, o una figlia vergine consacrata a Dio; non tenga in casa propria nessuna ché sia estranea (can. 27) .

Relativamente allo statuto funzionale si dice che coloro che sono stati battezzati da un diacono che regge una comunità che non ha vescovo o presbitero devono in seguito perfezionare il proprio battesimo mediante la benedizione del vescovo (can. 77) ${ }^{4}$. Il canone presenta il caso di una comunità retta da diacono ma il suo battesimo era dato in caso di necessità e comunque rimaneva la prerogativa del vescovo di amministrare i riti post-battesimali.

La questione del celibato per il vescovo, i presbiteri ed anche i diaconi diventò un elemento costante nella Chiesa latina. Tale disposizione era ribadita da una lettera di papa Siricio nel 385 che richiama sacerdoti e leviti alla "legge indissolubile" del celibato ${ }^{5}$.

${ }^{1}$ Cf. Per approfondire tale Concilio si veda: M. Sottomayor Muro, J. F. Ubiña, El Concilio de Elvira y su tiempo, Granada 2005, pp. 21-24; Ph. Badot, D. De Deckers, Historicité et actualité des canones disciplinaires du concile d'Elvire, "Augustinianum" 37 (1997), pp. 311-325.

2 Sinodo di Elvira, in: Enchiridion fontium historicae Ecclesiasticae Antiquae, ed.

C. Kirch, Barcellona 1973, n. 339.

${ }^{3}$ Ibidem, n. 336.

${ }^{4}$ Cf. ibidem, n. 401.

${ }^{5}$ Siricio, Littera directa ad decessorem ad Himerium tarraconensem epi- 
Il Concilio di Toledo del 400 accusava che la norma non trovava applicazione universale. Infatti il canone primo chiedeva che i diaconi dovevano vivere celibi, e se uxorati, dovevano vivere in castità e continenza ${ }^{6}$.

Anche il Concilio di Orange I del 447, disponeva che non dovevano essere ordinati diaconi coloro che erano coniugati, a meno che non decidevano di vivere in castità (can. 22); e in caso di mancato rispetto della norma dovevano essere espulsi dall'ufficio (can. 23) 7 .

Il Concilio di Agde del 506 che prescriveva l'età minima dell'ordinazione fissandola a 25 anni disponeva che i giovani coniugati potevano chiedere di essere ordinati col consenso della moglie poiché doveva cessare in tutto la vita coniugale ${ }^{8}$.

Il Concilio di Toledo del $527^{9}$ decretava che i bambini offerti dai genitori per il servizio del clero, dopo la tonsura, dovevano essere educati sotto la responsabilità del vescovo (can. 1, 1); ma, raggiunta l'età di diciotto anni dovevano essere interrogati dal vescovo, davanti al clero e al popolo, sulla loro intenzione circa il matrimonio, se per ispirazione divina si decidono per la grazia della castità e si obbligano alla professione del celibato, a vent'anni dovevano ricevere il suddiaconato (can. 1,2). A venticinque anni dovevano ricevere il diaconato (can. 1, 3); se, nonostante l'impegno contraevano le nozze, dovevano essere condannati come sacrileghi, e considerati esterni alla Chiesa (can. 1, 4).

scopum, 10 febbraio 385, in: Enchiridion symbolorum, ed. H. Denzinger, n. 185, pp. 103-104.

${ }^{6}$ Cf. Concilio di Toledo, in: Enchiridion fontium historicae Ecclesiasticae Antiquae, ed. C. Kirch, Barcellona 1973, n. 707.

7 Cf. Concilio di Orange, in: Enchiridion fontium historicae Ecclesiasticae Antiquae, ed. C. Kirch, Barcellona 1973, nn. 871-872.

${ }^{8}$ Cf. Concilio di Adge, in: Enchiridion fontium historicae Ecclesiasticae Antiquae, ed. C. Kirch, Barcellona 1973, nn. 963-966. Il Concilio, rilevava il problema di diaconi e presbiteri coniugati che avevano ripreso i rapporti coniugali. Esso si riferisce ad una lettera indirizzata a Eusebio Vescovo di Tolosa del 405, di papa Innocenzo I, il quale si richiamava all'autorità di papa Siricio in merito al celibato sacerdotale. Siano privati di ogni dignità ecclesiastica coloro che posti in tali uffici (presbiterale e diaconale), si sono comportati da incontinenti; né si conceda di accedere ad un ministero che può essere esercitato solo nella continenza (ibidem, n. 963).

9 Cf. K. Baus, Storia della Chiesa tra oriente e occidente, Milano 1992, pp. 274-275. 
Quanto a coloro che al tempo dell'interrogatorio avevano espresso la volontà di sposarsi, non possiamo certamente togliere la facoltà concessa dagli apostoli: costoro, dopo essersi sposati, potranno aspirare di salire i gradini sacri quando, in età più matura, avranno promesso, di comune accordo, di rinunciare alle opere della carne $(\text { can. } 1,5)^{10}$.

Per quanto attiene la posizione assunta in Oriente, sono interessanti le disposizioni di due Concili: quello di Ancira e il Quinisesto.

Il Concilio di Ancira, del 314 stabiliva che i diaconi, che nella propria ordinazione avevano manifestato l'intenzione di sposarsi, continuavano ad esercitare il ministero anche dopo il matrimonio, poiché avevano ottenuto il permesso dal vescovo. Coloro che invece nella ordinazione avevano taciuto professando quindi di rimanere celibi, non potevano accedere al matrimonio e se l'avessero fatto dovevano lasciare il ministero (can. 10) ${ }^{11}$.

Il Concilio Quinisesto o Trullano II del $692^{12}$, si poneva in posizione fortemente polemica contro la Chiesa di Roma; chiedeva che suddiaconi, diaconi e presbiteri di vivessero regolarmente la vita coniugale:

Poiché abbiamo saputo che nella Chiesa romana si ritiene come canone trasmesso per tradizione (la regola) di promuovere al diaconato o al presbiterato solo coloro che si impegnano a non avere più rapporti coniugali con le proprie mogli, noi, fedeli allantico canone della disposizione e della perfezione apostolica, vogliamo che rimanga stabile e legittimo il coniugio degli uomini sacri ${ }^{13}$.

${ }^{10}$ Concilio di Toledo, in: Enchiridion fontium historicae Ecclesiasticae Antiquae, n. 974. La disposizione risulta essere particolarmente interessante in quanto lascia suppore la presenza di una casa di formazione nella quale i giovani venivano educati sotto la responsabilità del vescovo. Questi primi esempi di luoghi formativi potrebbero essere individuati come l'inizio di quello che nel passare dei secoli assumerà la forma del seminario ed in modo particolare del seminario minore.

${ }^{11}$ Cf. Concilio di Ancira, in: Enchiridion fontium historicae Ecclesiasticae Antiquae, ed. C. Kirch, Barcellona 1973, n. 381.

12 Cf. O. Bucci, P. Piatti, Storia dei concili ecumenici, Roma 2014, p. 235.

13 Concilio Quinisesto, in: Enchiridion fontium historicae Ecclesiasticae Antiquae, ed. C. Kirch, Barcellona 1973, n. 1093. 
Si disponeva la deposizione per coloro che impedivano la vita coniugale ai presbiteri, diaconi e suddiaconi così pure ai presbiteri, diaconi e suddiaconi che si separavano dalla moglie col pretesto della pietà (can. 13). Questo Concilio contesta la richiesta della continenza per coloro che sono ordinati ministri nonostante fossero già ammogliati ${ }^{14}$.

Vari Concili si preoccuparono delle prerogative sacramentali dei diaconi.

Il Concilio Toletano dellanno 400, per esempio, stabiliva che in mancanza del vescovo, o se in sua presenza fosse stato delegato, solo il presbitero poteva cresimare, e non il diacono (can. 20) ${ }^{15}$.

Relativamente all'Eucaristia nei Concili antichi, sovente troviamo ingiunzioni disciplinari che proibiscono ai diaconi di celebrare l'Eucaristia o che diffidano da altri tipi di abuso.

A tale riguardo il Concilio di Arles I del 314 si esprimeva:

Quanto ai diaconi, ci è pervenuta notizia che in molte parti celebrano l'Eucaristia; venne deciso che ciò non deve succedere per nessuna ragione $(\text { can. 15) })^{16}$.

Tale disposizione registra il periodo di massimo splendore del diaconato.

Anche nel Concilio di Nicea del $325^{17}$ vi è traccia del dato che i diaconi cercassero di accrescere le proprie prerogative ampliando il loro statuto:

${ }^{14}$ Cf. ibidem, nn. 1094-1095.

15 Cf. Concilio di Toledo, in: Enchiridion fontium historicae Ecclesiasticae Antiquae, n. 712. Il Concilio stabiliva anche l'espulsione a presbiteri, diaconi e suddiaconi che non partecipavano alla messa tutti i giorni, quando abitano in un paese dove esiste la chiesa (can. 5); proibiva lordinazione diaconale a colui che dopo il battesimo avesse fatto il soldato (can. 8); chiedeva all'arcidiacono di ricordare sempre le leggi emanate dal Concilio (can. 20) in modo che il vescovo poteva custodirle e i presbiteri non le trascurassero (cf. ibidem, nn. 708-709).

${ }^{16}$ Concilio di Arles I, in: Enchiridion fontium historicae Ecclesiasticae Antiquae, ed. C. Kirch, Barcellona 1973, n. 373.

${ }^{17}$ Cf. W. A. Bienert, Das vornicänische homoousios als Ausdruck der Rechtgläbigkeit, "Zeitschrift für Kirchengeschichte" 90 (1979), pp. 5-29; K. Schatz, Storia dei concili. La Chiesa nei suoi punti focali, Bologna 1999, pp. 21-31; W. Gessel, Nizäa 325: Die Doppelstruktur eines Reichskonzils, in: Synodale Strukturen der Kirche, Donauwörth 1977, pp. 61-77. 
È pervenuta notizia al santo e grande Concilio che in alcuni luoghi e città i diaconi danno la Comunione ai presbiteri, ciò che non è permesso da nessun canone né dalla consuetudine, che cioè coloro che offrono il corpo di Cristo lo abbiano a ricevere da coloro che non hanno il potere di offrirlo. Così come sappiamo che alcuni diaconi, in presenza del vescovo prendono da sé l'eucaristia. Di fronte a tutto ciò vogliamo che i diaconi rimangano al proprio posto, convinti che sono al servizio del vescovo e inferiori ai presbiteri. Ragion per cui i diaconi facciano la Comunione dopo i presbiteri, ricevendola o dal vescovo o dal presbitero. E ancora, non è lecito al diacono sedere fra i presbiteri se ciò avviene è contro i canoni e il giusto ordine. Colui che non vorrà adeguarsi a queste regole deve lasciare la diaconia ${ }^{18}$.

\section{I CONCILI DEL V E VI SECOLO}

Le decisioni conciliari del V e VI secolo spesso furono una reazione agli abusi avvenuti nella vita della Chiesa. Pertanto, tali disposizioni non riflettono tutta la realtà della Chiesa e spesso il quadro normativo risultava essere più complesso di quanto costituito da norme precedentemente promulgate e da consuetudini.

Un primo dato che appare è il tema del celibato. La richiesta della vita nella continenza dopo l'ordinazione, espressa dai Concili, non significava che in quest'epoca i candidati al clero si reclutassero solo tra i celibi. Ancora alla fine del quinto secolo il candidato al diaconato poteva essere un uomo sposato e vivente nella famiglia a patto che dal momento dellordinazione rinunziasse alla vita coniugale e familiare. In questi termini si esprimeva il Concilio di Cartagine del $397^{19}$. Occorreva però una chiara dichiarazione di vita nella continenza da parte del candidato $^{20}$. La promessa doveva essere mantenuta sotto un accurato controllo della Chiesa. Tale obbligo era trattato spesso congiuntamente

18 Concilio di Nicea, Canones, in: Conciliorum oecumenicorum decreta, ed. J. Alberigo, P. Joannou, C. Leonardi, P. Prodi, Bologna 1991, nn. 13, 30-38; 14, 1-8.

${ }^{19}$ Cf. Concilio di Cartagine, cann. 11; 12; 13, in: Concilia Africae a. 345-525, ed. C. Munier, Corpus Christanorum Series Latina (CCSL 149), Turnhout 1974, p. 37.

${ }^{20}$ Cf. Concilio Arles, can. 43, in: Concilia Galliae a. 314-506, ed. C. Munier, Corpus Christanorum Series Latina (CCSL 148), Turnhout 2001, p. 122. 
a quello rivolto ai vescovi e ai presbiteri. Le direttive dei Concili richiedevano dal clero dal momento dellordinazione una rigida cautela nei contatti con tutte le donne. Ai chierici era anzitutto vietato di abitare con extraneis mulieribus ${ }^{21}$, ma gli era precluso ogni tipo di atteggiamento di tipo "familiare" con le donne non consanguinee. Il divieto toccava tutto il clero a gradu diaconatus ${ }^{22}$. La cautela nei contatti con le donne, tranne quelle appartenenti alla vicina parentela, era richiesta da tutti i membri del clero, diacono incluso, e le sanzioni in caso di disubbidienza furono severe. Una vita nel celibato, il divieto della familiarità con le donne ma anche la cautela con tutto ciò che riguardava tutto il contesto sessuale (per esempio le parole e le canzoni) erano visti come componenti della castità conveniente ad un uomo operante nellambiente sacro, dedicato al Signore. Un chierico non doveva neanche dare loccasione a male loquendi vel sentiendi ${ }^{23}$. Largomentazione che veniva citata in tutti questi casi era: un sacerdote ed un levita che divinis sacramentis inserviunt devono osservare la castità domandata da $\mathrm{Dio}^{24}$. La purezza sia del sacerdote sia del diacono, compresa in modo integrale, cioè del corpo e dello spirito, era indicata come una condizione della efficacia della loro preghiera ${ }^{25}$. Erano dunque le pure ragioni liturgiche e le funzioni di carattere sacro legate al culto che tracciavano il modello della vita del diacono. La santità delle funzioni che egli svolgeva domandava una vita nella continenza piena e insospettabile. Tale era almeno l'ideale proposto dalla Chiesa. Le disposizioni ed i decreti conciliari avevano la loro forza coercitiva attraverso le conseguenti pene da infliggere a coloro che non avessero osservato il precetto. I chierici colpevoli della familiarità e che in modo tenace non reagivano agli ammonimenti del vescovo dovevano essere allontanati dal

${ }^{21}$ Cf. Statuta Ecclesiae Antiqua, can. 27, in: Concilia Galliae a. 314-506, ed. C. Munier, Corpus Christanorum Series Latina (CCSL 148), Turnhout 2001, p. 171.

${ }^{22}$ Cf. Concilio Arles, can. 3, in: Concilia Galliae a. 314-506 (CCSL 148), p. 174.

${ }^{23}$ Cf. Concilio di Tours, can. 3, in: Concilia Galliae a. 314-506, ed. C. Munier, Corpus Christanorum Series Latina (CCSL 148), Turnhout 2001, p. 143.

${ }^{24}$ Cf. Concilio di Cartagine, cann. 11; 12; 13: in: Concilia Africae a. 345-525 (CCSL 149), p. 143.

${ }^{25}$ Cf. Concilio di Tours, can. 1, in: Concilia Galliae a. 314-506 (CCSL 148), p. 143 . 
servizio e privati della Comunione ${ }^{26}$. In vista dell'obbligo della continenza e del celibato non si promuoveva al clero e perciò anche al diaconato se non degli uomini che davano qualche garanzia di poter osservare questo dovere ${ }^{27}$. Generalmente un chierico, e quindi anche il diacono, doveva essere al di fuori di comportamenti riprovevoli. Per questa ragione il chierico doveva evitare dei luoghi di scarsa riputazione, per esempio le taverne, e soltanto nel caso di necessità era permesso frequentarli ${ }^{28}$. I chierici dovevano evitare le feste di nozze come pure ai banchetti con le danze e con le canzoni oscene ${ }^{29}$. Avevano altresì il divieto di partecipare ai banchetti preparati dai giudei per non provocare scandalo ${ }^{30}$. Il pericolo dello scandalo era legato anche all'ubriachezza, infatti tale costume sembrava essere assai diffuso tra i membri del clero. I Concili biasimavano il costume ma le pene erano previste soltanto nel caso del trascurare per motivo dell'ubriachezza il servizio liturgico. Un chierico colpevole di tale negligenza doveva essere allontanato per un congruo tempo dalla comunione ma poteva anche subire un supplicio corporale ${ }^{31}$, o essere punito con la perdita della sua fonte di sostentamento ${ }^{32}$. Come tutti i membri del clero il diacono doveva rispettare le norme riguardanti le occupazioni da svolgere. Le disposizioni conciliari non permettevano al chierico di dedicarsi a occupazioni che potevano esporre al danno la sua morale, ed inoltre, non doveva impegnarsi nelle occupazioni che rendevano impossibile oppure ostacolavano l'esecuzione dei doveri legati con l'incarico. Vietati erano dunque anzitutto le occupazioni che domandavano i frequenti viaggi come anche i mestieri che impegnavano tutto il tempo. Nel linguaggio ecclesiale si parlava delle occupazioni che distoglie-

${ }^{26}$ Cf. ibidem, can. 3, p. 143.

${ }^{27}$ Cf. Concilio di Adge, can. 9, in: Concilia Galliae a. 314-506, ed. C. Munier, Corpus Christianorum Series Latina (CCSL 148), Turnhout 2001, p. 197.

${ }^{28}$ Cf. Concilio di Cartagine, can. 26, in: Concilia Africae a. 345-525 (CCSL 149), p. 40.

${ }^{29}$ Cf. Concilio di Vannes, can. 11, Concilia Galliae a. 314-506, ed. C. Munier, Corpus Christanorum Series Latina (CCSL 148), Turnhout 2001, p. 154.

${ }^{30}$ Cf. ibidem, can. 11, p. 155.

${ }^{31}$ Cf. Concilio di Tours, can. 2, in: Concilia Galliae a. 314-506 (CCSL 148), p. 145; Concilio di Vannes, can. 13, in: Concilia Galliae a. 314-506 (CCSL 148), p. 155.

32 Cf. Statuta Ecclesiae Antiqua, can. 35, in: Concilia Galliae a. 314-506 (CCSL 148), p. 172. 
vano dai "doveri ecclesiali" 33 . Conforme ai decreti conciliari i chierici non dovevano impegnarsi negli affari di carattere economico. Generalmente i Concili biasimavano i mestieri legati direttamente con il denaro perché sempre creavano il sospetto d'agire per motivo di cupidigia ${ }^{34}$. Il chierico che si lasciava guidare dal desiderio del "guadagno iniquo", oppure che cercava una occupazione lucrativa, in modo particolare legata all'usura, incorreva nella perdita dell'ufficio ${ }^{35}$. Queste norme restarono fedeli ad una tradizione già nota nel terzo secolo. I Concili parlarono anche del vestiario, del modo di presentarsi, e delle occupazioni non lavorative: si raccomandava una certa moderazione, mettendo in rilievo che non tutto conveniva al chierico ${ }^{36}$. Il modo di vestirsi e il comportamento, senza sfoggio e senza guardare la moda, doveva rispettare la spiritualità dell'uomo dedicato al servizio divino ${ }^{37}$.

Riguardo alle funzioni specifiche del diacono, i Concili parlano relativamente poco. Fra i doveri indicati dalle direttive conciliari si distinguono anzitutto quelle riguardanti la liturgia. Come tutto il clero il diacono doveva prendere parte alle celebrazioni liturgiche. Soltanto una malattia poteva scusare il diacono da questo dovere ${ }^{38}$. Nella liturgia il diacono svolgeva le funzioni proprie ma poteva in casi determinati sostituire altri chierici, anzitutto i presbiteri. Si può osservare un interessante processo: la sfera dei compiti che il diacono poteva sbrigare in sostituzione del presbitero progressivamente cresceva anche se lentamente.

Le disposizioni contenute nelle disposizioni conciliari di questo periodo riguardavano spesso la partecipazione dei diaconi all'Eucaristia e gli abusi che si verificavano. Vi fu una autorizzazione dei diaconi a portare

${ }^{33}$ Cf. ibidem, can. 29, p. 171.

${ }^{34}$ Cf. Concilio di Cartagine, can. 15, in: Concilia Africae a. 345-525 (CCSL 149), p. 38.

${ }^{35}$ Cf. Concilio di Adge, can. 9, in: Concilia Galliae a. 314-506 (CCSL 148), p. 197.

${ }^{36}$ Cf. Furono vietati: portare la barba (cf. Statuta Ecclesiae Antiqua, can. 25, in: Concilia Galliae a. 314-506 [CCSL 148], p. 171); caccia con i cani (cf. Concilio di Adge, can. 8, in: Concilia Galliae a. 314-506 [CCSL 148], p. 226).

${ }^{37}$ Cf. Concilio di Adge, can. 20, in: Concilia Galliae a. 314-506 (CCSL 148), p. 202.

${ }^{38}$ Cf. Concilio di Cartagine, can. 20, in: Concilia Africae a. 345-525 (CCSL 149), p. 107. 
le albe durante oblationis et lectionis ${ }^{39}$. Il diacono leggeva il vangelo, dirigeva i fedeli, aiutava il vescovo durante la distribuzione della Comunione, a patto che ci fosse una necessità. Da quanto appare la distribuzione della Comunione non apparteneva ai suoi doveri ma era un privilegio derivante da una necessità ${ }^{40}$. All'inizio del VI secolo i Concili ricordavano che un diacono seppur sanzionato di una pena, nel caso di necessità e nell'assenza del presbitero poteva battezzare ${ }^{41}$. Restano dubbi se nella normalità il diacono potesse battezzare. Nei decreti del Concilio di Vaison dal $529^{42}$, si permetteva al diacono, nel caso in cui il presbitero fosse impossibilitato, di leggere durante l'Eucaristia un'omelia dei "santi padri" "33. Tale concessione sorgeva da necessità pastorali ma, secondo alcuni storici, rileva una certa agitazione dei diaconi che non erano soddisfatti dei diritti loro concessi e in tal senso presentavano pretese. Tale Concilio sancì la nascita delle scuole parrocchiali ${ }^{44}$, alle quali a partire da allora sarebbe stato affidato il compito della formazione non solo di chi avesse voluto abbracciare la vita ecclesiastica, ma anche di chi avesse voluto, pur restando laico, avere per sé una certa cultura. Fino a quell'anno era in vigore quanto stabilito dal II Concilio di Toledo del $527^{45}$, con cui si erano istituire le scuole vescovili, che di fatto esistevano già da qualche tempo. A Toledo non si era fatto altro che sancire che presso l'abitazione del vescovo si sarebbero potuti radunare dei giovani, che sarebbero stati istruiti nella teologia e nelle

39 Cf. ibidem, can. 41, p. 348.

${ }^{40}$ Cf. Statuta Ecclesiae Antiqua, can. 58, in: Concilia Galliae a. 314-506 (CCSL 148), p. 176; Concilio di Cartagine, can. 29, in: Concilia Africae a. 345-525 (CCSL 149), p. 348 .

${ }^{41}$ Cf. Concilio di Orléans, can. 8, in: Concilia Galliae a. 511-695, ed. C. De Clercq, Corpus Christianorum Series Latina (CCSL 148 A), Turnhout 1963, p. 8.

${ }^{42}$ Si trattò di un Concilio molto ristretto quanto a numero di partecipanti (appena 11 o 12), ma tale da rivestire un ruolo di capitale importanza nella storia della cultura e della scuola medievali (cf. G. Andenna, Pievi e parrocchie in Italia centro-settentrionale, in: Pensiero e sperimentazioni istituzionali nella Societas Christiana, 1046-1250. Atti della sedicesima Settimana internazionale di studio, Mendola, 26-31 agosto 2004, a cura di G. Andenna, Milano 2007, pp. 370-373.

${ }^{43}$ Cf. Concilio di Vaison, can. 2, in: Concilia Galliae a. 511-695, ed. C. De Clercq, Corpus Christianorum Series Latina (CCSL 148 A), Turnhout 1963, p. 79.

${ }^{44}$ Cf. ibidem, can. 1, p. 78.

${ }^{45}$ Cf. K. Baus, Storia della Chiesa tra oriente e occidente, pp. 272-273. 
lettere da un apposito maestro, lo scolasticus. Ancor prima del Concilio di Toledo, nella città di Arles, il vescovo Cesario ${ }^{46}$ aveva già realizzato un tipo di coabitazione tra chierici che prevedeva, oltre alla preghiera, la lettura privata e l'ascolto comunitario delle Sacre Scritture (per coloro che aspiravano al diaconato era prescritta la lettura per almeno quattro volte di seguito del Vangelo e del Nuovo Testamento). Tuttavia, come si comprende bene, questo tipo di istituzione scolastica non riusciva a raggiungere un ampio numero di persone. E questo perché le sedi episcopali sorgevano solo nelle grandi città, e dunque venivano tagliate fuori dal provvedimento di Toledo tutte le realtà geografiche non solo rurali, ma anche quelle caratterizzate dalla presenza di piccoli centri abitati. A questo le disposizioni del Concilio di Vaison posero rimedio ${ }^{47}$, inoltre il sorgere di queste scuole, che prendevano il posto di quelle municipali romane, ormai del tutto scomparse, rappresentano insieme con quelle che andavano nascendo all'interno dei monasteri, la base della cultura europea medievale. In tale contesto si può leggere la disposizione che permise ai diaconi la lettura delle omelie dei santi Padri, qualora nelle parrocchie e non solo quelle cittadine, il presbitero non potesse predicare per motivi di salute. Questo perché, come si legge nel canone, se i diaconi hanno il diritto di leggere la parola di Dio durante gli uffici sacri, e ne sono dunque degni, come pensare che non lo siano relativamente alle omelie dei Padri? ${ }^{48}$.

Altre proibizioni, che si ritrovano in questo periodo, rilevano una certa rivalità tra i presbiteri e i diaconi come per esempio il divieto della distribuzione della Comunione ai diaconi prima dei presbiteri ${ }^{49}$, oppure il divieto della benedizione del calice e della distribuzione del pane ${ }^{50}$.

${ }^{46}$ Cf. P. A. Février, Césaire et la Gaule méridionale au VI siècle, in: Césaire d’Arles e la Christianisation de la Provence. Actes des journées "Césaire» (Aix-en-Provence-ArlesLérins, 3-5 novembre 1988, 22 avril 1989), a cura di D. Bertrand, Paris 1994, pp. 45-73.

47 Cf. Concilio di Vaison, can. 2, in: Concilia Galliae a. 511-695 (CCSL 148 A), p. 78 .

${ }^{48}$ Cf. M. Morichetti, La predicazione di Cesario di Arles al superstizioso, in: Evangelizzazione dell'Occidente dal terzo all'ottavo secolo, a cura di L. Bacci, I. Mazzini, Roma 2001, pp. 105-123.

${ }^{49}$ Cf. Concilio di Cartagine, can. 108, in: Concilia Africae a. 345-525 (CCSL 149), p. 296.

${ }^{50}$ Cf. G. Hammann, Storia del diaconato, Magnano 2004, pp. 84-85. 
Il silenzio dei Concili sugli altri doveri e funzioni non esclude che i diaconi abbiano avuto altri compiti. Più probabile nelle funzioni liturgiche, viste il sorgere di nuove modalità di espressione o abusi, sorgeva il bisogno delle normative stabilite dai Concili. Un dato chiaro è che il diacono era visto come lassistente del vescovo che lo poteva incaricare di altri compiti nel campo di cui era responsabile, primi tra tutti il campo caritativo. Infatti spesso tali Concili ricordavano ai vescovi il dovere della protezione dei poveri. Possibile che il suo delegato in questo campo fosse un diacono.

\section{L'ALTO MEDIOEVO}

Nell'Alto Medioevo la presenza del diacono nelle comunità ecclesiali assunse un'ulteriore evoluzione. Il diacono essendo esecutore del vescovo in materia di carità, i compiti di cui l'episcopato era spesso gravato si riversarono su di lui con non poche situazioni di abuso di potere spesso riprovate dai Concili ${ }^{51}$. Questo periodo fu caratterizzato da una carenza nel servizio diaconale, ragione per cui altre componenti della comunità cristiana come laici, nobili, senatori, svolsero le attività occupandosi e di operare la carità ${ }^{52}$. In questo periodo assistiamo all'impegno di non chierici che organizzano e operano una vera diaconia.

Questo non significa che i chierici si disinteressarono della vicinanza ai poveri. Infatti, alla fine del VI secolo il papa Gregorio Magno (590-604), egli stesso già monaco e già diacono, col cui pontificato si chiude il periodo patristico, si sforzò di restituire a questo aspetto del ministero della Chiesa la sua funzione caritativa. Presentandosi come servus servorum Dei, nel 595 scrisse a un diacono di nome Cipriano:

Zenone, fratello e coepiscopo nostro, $\mathrm{mi}$ fa sapere che gli abitanti della sua città soffrono per la mancanza di alimenti. Poiché desideriamo aiutarli, come è possibile, ti prescriviamo di dare al predetto nostro fratello e coepiscopo mille moggi di grano $\mathrm{e}$, se ne potrà caricare di più, anche sino a duemila. Ti esortiamo quindi a non frapporre indugio e scusa

\footnotetext{
${ }^{51}$ Cf. ibidem, pp. 117-118.

${ }^{52}$ Cf. ibidem, pp. 105-117.
} 
a queste prebende, perché egli, fin quando il tempo lo permetta, con l'aiuto di Dio, possa tornare alla propria chiesa e sovvenire rapidamente a coloro che sono nel bisogno ${ }^{53}$.

La lettera dimostra l'afflato caritativo che anima i pastori, ma essi non disponevano dei mezzi per poter esercitare la loro funzione caritativa in maniera efficace. Infatti, la suddivisione del compito tra il vescovo, che aveva la responsabilità caritativa, e il diacono, che ne era l'esecutore, contribuiva fortemente a rendere inefficace l'esercizio della carità nelle miserie dell'epoca. Divenuti dei semplici amministratori, i diaconi persero il loro fondamento teologico. Spesso si verificarono situazioni in cui essi fecero i propri interessi con le risorse che il vescovo affida loro. Così come, anche i vescovi, si appropriarono indebitamente di beni che erano destinati alla carità ${ }^{54}$. Di questo ne abbiamo traccia, per esempio, in un'altra lettera di Gregorio Magno, scritta verso il 596, e indirizzata al vescovo Protasio:

Affinché la buona stima che l'opinione comune ha di voi possa essere confermata dai fatti, dite al nostro fratello e coepiscopo Virgilio, che si adoperi a restituirci le somme di denaro che il suo predecessore ha ricevuto da parte nostra per molti anni e ha trattenuto presso di sé, poiché sono beni dei poveri. Insistete che non trattenga presso di sé i beni di Pietro e dei poveri ${ }^{55}$.

La lettera testimonia l'auto comprensione di Gregorio Magno di ritenersi amministratore dei beni dei poveri, in quanto Vescovo di Roma. Egli si considerava sempre come sorvegliante del compito diaconale. Progressivamente i beni di cui la comunità disponeva, furono destinati al sostentamento del clero e alle esigenze della comunità stessa. Cominciò a mancare un riferimento ministeriale per vegliare sul soccorso ai poveri, e per organizzarlo nelle condizioni di emergenza. Il loro compito di gestire la parte dei beni destinati all'azione caritativa portò varie situazioni, spesso incresciose nell'amministrazione. Questo va contestualizzato nell'ascesa dei

${ }^{53}$ Gregorius PP. I, Lettere VI, 4, ed. J. P. Migne, Patrologiae Cursus Completus. Series Latina (in seguito: PL), 77, Parisiis 1862, p. 796.

${ }^{54}$ Cf. G. Hammann, Storia del diaconato, pp. 122-123.

${ }_{55}$ Gregorius PP. I, Lettere VI, 55, in: PL, pp. 839-840. 
re franchi e nella rivalità che questi avevano nei confronti dei vescovi ${ }^{56}$. Il conflitto si concentrò intorno al problema delle imposte, eredità, ancora una volta, del mondo romano al tramonto ${ }^{57}$. Mentre il diacono aveva il compito di distribuire l'imposta ai poveri, il vescovo doveva vigilare affinché il fondo fosse regolarmente alimentato mediante la decima. Questo provocò non poche ostilità tra vescovi e diaconi, finché nell'VIII secolo le autorità imperiali e regie ristabilirono l’obbligo di pagare la decima nella loro legislazione regale ${ }^{58}$. Il vescovo divise l'imposta in quattro parti: una per se stesso, una per il suo clero, una per il mantenimento delle chiese e una per i poveri. Il diacono amministrava quest'ultima parte, secondo gli ordini ricevuti dal vescovo. La parte destinata alla carità variava secondo la generosità dei vescovi ${ }^{59}$.

Un altro fenomeno fu la progressiva moltiplicazione delle chiese che servivano territori rurali ${ }^{60}$. Così la gestione delle risorse fu orientata verso tali organizzazioni e non legata ai diaconi. In tali situazioni la carità fu gestita da laici o dagli ordini religiosi ${ }^{61}$.

Pertanto si può dire che il diacono mantenne la sua partecipazione liturgica subalterna, determinata dall'autorità del vescovo; a questo titolo, era indispensabile alla vita cultuale delle chiese. Si affievolì il compito caritativo a favore di quello propriamente gestionale dei beni, aiutava il vescovo, e sostituiva in modo sussidiario il presbitero nelle campagne.

\section{IL MEDIOEVO}

Questo fu il periodo che vide l'ascesa della dinastia carolingia (751-814) e dell'impero franco (814-843). Si costituì un nuovo sistema

${ }^{56}$ Cf. I. Hertling, A. Bulla, Storia della Chiesa. La penetrazione dello spazio umano ad opera del cristianesimo, Roma 2001, pp. 154-157.

${ }^{57}$ Cf. G. Hammann, Storia del diaconato, p. 122.

${ }^{58}$ Cf. H. Pirenne, Les villes du Moyen Áge, essaì d'histoire économique et sociale, Paris 1927, pp. 43-44.

59 Cf. G. Hammann, Storia del diaconato, p.123.

${ }^{60}$ Cf. E. Cruzel, Lorganizzazione ecclesiastica nelle campagne, "Reti medievali" 1 (2010), pp. 417-435.

${ }^{61}$ Cf. E. Pitz, Europäisches Städtewesenund Bürgertum von der Spätantike bis zum holen Mittelalten, Darmastadt 1991, pp. 151-155. 
rurale e agricolo: unità formate dalla casa e dalle terre coltivabili, con diritti di uso sulle foreste e sui prati, si raggruppano intorno e sotto l'autorità delle signorie feudali. Anche il Vescovo di Roma, e in generale i vescovi strinsero accordi con la dinastia franca. Dopo l'incoronazione di Pipino a Soissons nel 751, primo re consacrato con l'unzione dell'olio santo ${ }^{62}$, l'autorità regale fu investita di un potere divino. Nell'anno 800, Carlo Magno, incoronato nella basilica di San Pietro a Roma come imperatore del Sacro Romano Impero, divenne tutore della Chiesa e, a questo titolo, poté intervenire nellorganizzazione e nei conflitti dottrinali dell'autorità ecclesiastica ${ }^{63}$. Tuttavia questa organizzazione portò al futuro conflitto tra il potere politico e l'autorità religiosa riguardo alla suprema sovranità sulla società del tempo. Nel IX secolo s'impose la feudalità di tipo franco. Guerrieri che si erano distinti ricevettero dal principe concessioni territoriali, sulle quali si stabilirono nella reciprocità dei rapporti di fedeltà, e di diventare cavalieri. I diaconi ordinati continuarono a mantenere le nuove chiese ${ }^{64}$.

La figura diaconale fu assunta come modello da parte degli stessi sovrani ${ }^{65}$. Carlo Magno si riconosceva investito della suprema responsabilità in materia di diaconia caritativa ${ }^{66}$. L'imperatore si sentiva investito del servizio caritativo mentre il diacono chierico, prossimo del vescovo, era relegato nel solo compito liturgico e amministrativo. Un brano del discorso di intronizzazione, di Carlo Magno, conservato nei capitolari (i decreti imperiali), attesta la volontà di riformare la funzione e il ministero diaconali:

Amate il vostro prossimo come voi stessi e date ai poveri l'elemosina con tutto il cuore. Accogliete gli stranieri nelle vostre case, visitate i malati

${ }^{62}$ Cf. G. Gandino, Contemplare l'ordine: intellettuali e potenti dell'alto Medioevo, Napoli 2004, pp. 19-21; J. Chidenius, Medieval institutions and the Old Testament, Helsinki Helsingfors 1965, pp. 44-47.

${ }^{63}$ Cf. R. Scheinder, Karl der Grosse, politisches Sendungsbewusstein und mission, in: Kirchengeschichte als Missiongeschichte, II, a cura di H. Frohnes, H. W. Gensichen, G. Kretschmar, München 1978, pp. 227-248.

${ }^{64}$ Cf. R. Barbieri, Uomini e tempo medievale, Milano 1988, pp. 298-299.

${ }^{65}$ Cf. G. Hammann, Storia del diaconato, pp. 131-132.

${ }^{66}$ Cf. W. Maurer, Die christliche Diakonie im Mittelalter, in: Das diakonische Amt der Kirche, a cura di H. Krimm, Stuttgart 1965, p. 137. 
e abbiate pietà di coloro che sono in prigione. Riscattate i prigionieri, sostenete coloro che sono ingiustamente oppressi, difendete le vedove e gli orfani ${ }^{67}$.

Carlo Magno, sin dalla sua intronizzazione, esplicitò la volontà di restituire al cristianesimo occidentale la sua originaria dimensione caritativa ricordando ai suoi sudditi, ossia ai battezzati che gli erano sottomessi, la loro responsabilità collettiva in questo campo. Egli si erse a primo "Diacono" della Chiesa in Occidente. Il suo biografo, Eginardo (770-840), pose in evidenza questa pretesa imperiale:

Per quanto riguarda la cura dei poveri e la loro assistenza mediante elemosine, Carlo diede prova di un grande zelo e di molta pietà; e questo non soltanto nel suo paese e nel suo impero, ma anche in regioni al di là dei mari, ove egli aveva l'abitudine di inviare delle somme di denaro: in Siria, in Egitto e in Africa, a Gerusalemme, ad Alessandria, a Cartagine, quando veniva a sapere che là c'erano dei cristiani bisognosi. E' proprio questo il motivo per cui egli si adoperò per guadagnare l'amicizia dei re di quelle regioni al di là del mare, al fine di poter venire in aiuto ai cristiani che vivevano sotto il loro dominio ${ }^{68}$.

Questo fa comprendere come, se da un lato il ministero diaconale ordinato non sia stato una presenza determinante, nella società la difesa del povero fosse considerato un tratto di nobiltà d'animo, elemento necessario per mettere in risalto la figura imperiale per conferire prestigio maggiore a questi nei confronti dellautorità ecclesiastica nel contesto che successivamente assunse i contorni della lotta per le investiture. Infatti nel diritto franco-germanico si conferì al signore feudale (laico) la qualifica diaconale di protector et defensor dei bisognosi. Il signore tuttavia, in quanto laico, doveva disporre di un minimo di infrastruttura per poter materialmente aiutare gli indigenti del suo feudo. Questo non sempre era realizzato, e spesso implementato in luoghi di fortuna e con ricoveri di passaggio ${ }^{69}$.

67 Capitularia I, in: Monumenta Germaniae historica, I, a cura di A. Boretius, Hannoverae 1883, p. 239.

${ }^{68}$ Eginardo, Vita Karoli Magni, cap. 3, in: Sources d'histoire médiévale. IX-XIV siècle, a cura di G. Brunel, E. Lalou, Paris 1992, p. 23.

${ }^{69}$ Cf. G. Hammann, Storia del diaconato, pp. 134-135. 
Lo spirito diaconale prende forma nel sistema feudale attraverso un duplice canale: laicale ed ecclesiale. Quello laicale attraverso la responsabilità dei principi o dei signori, e quella ecclesiale attraverso il sorgere dei monasteri. Questo luogo ecclesiale diverrà nel medioevo luogo di formazione, cultura e soprattutto modello esemplare per la vita di carità ${ }^{70}$.

Questo movimento fa scorgere la perdita della prerogativa dell'amministrazione della carità nelle mani di un ministro preposto a questo a favore di tutta la comunità che si riscoprì chiamata ad una vocazione di servizio, attenzione all'indigente, al pellegrino, vocazione diaconale appunto.

È possibile constatare pertanto che nel Medioevo il diaconato ordinato non ha partecipato al rinnovamento della funzione caritativa che animò questo periodo storico.

Nei secoli XI e XII nacquero comunità come i francescani ${ }^{71}$, i domenicani ${ }^{72}$, e anche gli ordini "ospedalieri"73: templari, ordine di San Giovanni, ordine dei cavalieri teutonici ${ }^{74}$. Questi ordini si fecero portavoce di una crescente sollecitudine verso i malati e i meno abbienti che in questo periodo maturò nelle comunità ecclesiali. La povertà e la mendicità vennero considerate come un valore meritorio di alta autenticità e spiritualità cristiana $^{75}$. Questa dimensione teologica e spirituale spiega lo sviluppo che ebbero gli ospizi e gli ospedali dove venivano accolti malati e poveri ${ }^{76}$. La loro marginalizzazione e la loro solitudine, cioè la loro esclusione dalla società, appariva a poco a poco come un rinnegamento e persino un tradimento nei confronti di Dio. A questo proposito, il lebbroso era l'imma-

${ }^{70}$ Cf. ibidem, pp. 145-148.

${ }^{71}$ Cf. M. Bartoli, W. Block, A. Mastromatteo, Storia della spiritualità francescana. I - Secoli XIII-XVI, Bologna 2017, pp. 423-431.

${ }^{72}$ Cf. D. Penone, I domenicani nei secoli. Panorama storico dell'Ordine dei frati predicatori, Bologna 1998, pp. 56-62.

${ }^{73}$ Cf. M. Piana, C. Carlsson, Archaeology and Architecture of the Military Orders: New Studies, London 2016, pp. 134-201.

${ }^{74}$ Cf. A. Demurger, Chevaliers du Christ. Les Ordres religieux-militaires au Moyen Áge (XI e-XVI e siècle), Paris 2002, pp. 43-47.

${ }^{75}$ Cf. G. G. Merlo, Esperienze religiose e opere assistenziali nei secoli XII e XIII, Torino 1987, pp. 12-16.

${ }^{76}$ Cf. G. Chittolini, G. Miccoli, La Chiesa e il potere politico dal Medioevo all'età contemporanea, Torino 1986, pp. 147-193. 
gine più stupefacente del povero, malato cronico, contagioso, inguaribile, irrecuperabile per la società; da qui la sua esclusione simboleggiata dai campanelli che gli pendevano al collo per avvertire della sua vicinanza ${ }^{77}$.

L'esempio più conosciuto è certamente Francesco d'Assisi e le sue fraternità. È nota l'importanza determinante che la diaconia caritativa riviveva alla nascita di questa nuova struttura comunitaria: lo stesso Francesco voleva essere diacono per eccellenza, trovando in ogni malato - nei lebbrosi in particolare - l'incarnazione del Cristo sofferente e crocifisso. Nella concezione propria del "Poverello", il povero in genere riacquistava il senso teologico che il Cristo dei Vangeli gli aveva dato, quello di essere il destinatario privilegiato della misericordia divina ${ }^{78}$.

Per quanto riguarda il diacono ordinato, restava un ministero subalterno e si andava consolidando tale ministero come "scalino" di accesso al sacerdozio, legato ai compiti liturgici e amministrativi del vescovo.

La funzione della diaconia nella sua dimensione caritativa trovava in questo momento storico la fonte del rinnovamento in un'altra forma di vita cristiana regolare, attraverso le modalità menzionate del monastero, degli ordini mendicanti e degli ordini religiosi, che avevano il compito di far risvegliare la responsabilità diaconale di tutto il popolo cristiano dei battezzati ${ }^{79}$. Infatti, nella predicazione e nella teologia si fece spazio l'importanza delle opere di carità e misericordia, che offrivano molteplici possibilità ai bisogni di santificazione mediante le opere. Se da un lato la vita di carità, di servizio e quindi di diaconia del popolo di Dio era esaltata, non lo era relativamente alle funzioni diaconali propriamente dette.

Leggendo Tommaso, si nota come egli non attribuisca nessuna importanza al diacono in quanto ministro ordinato. Nella "Summa theologica", solo due volte parla dei diaconi, per indicare, in linea con la teologia, le disposizioni ereditate dalla tarda antichità:

Al diacono, come prossimo allordine sacerdotale, spettano alcuni compiti di tale ufficio, ossia la facoltà di dispensare il sangue (cioè di porgere il calice dell'Eucaristia): però non quella di dispensare il corpo, se non in

77 Cf. J. Agrimi, C. Crisciani, Malato, medico e medicina nel medioevo, Torino 1980, pp. 24-28.

${ }^{78}$ Cf. J. Le Goff, San Francesco d'Assisi, Roma-Bari 2000, pp. 105-107.

${ }^{79}$ Cf. G. Hammann, Storia del diaconato, pp. 157-158. 
caso di necessità, dietro comando del vescovo o del presbitero. Primo, perché il sangue di Cristo è contenuto nel calice, quindi non è a contatto di chi lo distribuisce, come invece il corpo di Cristo. Secondo, perché il sangue significa la redenzione che deriva al popolo da Cristo: tanto che al sangue viene mescolata dell'acqua per indicare il popolo. Ora, poiché i diaconi stanno tra il presbitero e il popolo, ai diaconi si addice di più la distribuzione del sangue che la distribuzione del corpo. Terzo, come il diacono partecipa un poco della virtù illuminativa del presbitero in quanto dispensa il sangue, così il presbitero partecipa del governo effettivo del vescovo, in quanto dispensa l'eucaristia che perfeziona l'uomo in se stesso unendolo a Cristo. Invece gli altri perfezionamenti che dispongono l'uomo in rapporto al prossimo sono riservati al vescovo ${ }^{80}$.

Nella concezione di Tommaso d'Aquino del diacono ordinato e delle sue funzioni, non vi sono delle grandi modifiche rispetto alla tarda antichità. Egli, scrivendo nella metà del XIII secolo, non fa alcun accostamento tra il ministero ordinato del diacono ${ }^{81}$, la povertà del suo compito caritativo e la comparsa dell'attenzione caritativa sorta presso le comunità monastiche e religiose. Viene ribadito che lui è "ministro del sangue" per una ragione puramente di subalternità rispetto al sacerdote, presbitero o vescovo, sia sacramentale che di partecipazione al governo del vescovo ${ }^{82}$. Il suo servizio è sottolineato soprattutto nella sinassi eucaristica e riguardante comunque solo l'ambito liturgico. La sua presenza è giustificata dall'essere prossimo all'ordine sacerdotale e non in riferimento ad un suo statuto proprio ${ }^{83}$.

\section{LE DISPOSIZIONI DEI CONCILI LATERANENSI}

Nel periodo del Medioevo non esistono quasi interventi canonici che riguardano direttamente il diaconato. Gli unici di cui abbiamo tracce

80 Thomae Aquinatis, Summa Theologiae III, 67, 82, 3-4, Madrid 1958, pp. 545-546.

${ }^{81}$ Cf. J. Rigali, Découvrir les ministèrs, Paris 2001, pp. 83-93; E. Castellucci, Il ministero ordinato, Brescia 2002, pp. 156-160.

${ }^{82}$ Cf. W. Kasper, Nocchiero in mezzo alla tempesta. Il ministero episcopale secondo Tommaso d'Aquino, in: Teologia e Chiesa, a cura di W. Kasper, Brescia 2001, pp. 109-135.

${ }^{83}$ Cf. F. Massagrande, Il Sacramento dell'Ordine: il presbiterato nella «Summa Theologiae» di S. Tommaso d’Aquino, "Esperienza e Teologia” 24 (2008), pp. 97-102. 
sono quelli del XII secolo nei Concili Lateranensi ${ }^{84}$. Il dato che non abbiamo molte disposizioni, potrebbe essere interpretato come una perdita di rilevanza di tale ministero. Tale situazione provocò pochi problemi e conseguentemente poche disposizioni di vescovi, pontifici e concili.

Il Concilio Lateranense I ${ }^{85}$, del 1123 , ordinò:

Assolutamente nessun arcidiacono o arciprete o prevosto o decano assegni la cura d'anime o le prebende della chiesa senza il giudizio o il consenso del vescovo, che anzi, come è stabilito dai sacri canoni, la cura delle anime e la distribuzione delle cose ecclesiastiche, rimangano nel giudizio e nel potere del vescovo. Chi agirà contro di ciò e vorrà arrogarsi un potere che è del vescovo, sia cacciato dai confini della Chiesa ${ }^{86}$.

La prassi riprovata testimonia dell'abuso che consumavano gli arcidiaconi di nominare sacerdoti da assegnare a delle chiese. Colui che occupava tale ufficio ecclesiastico, era innanzitutto l'amministratore di beni della Chiesa, pertanto probabilmente, qualcuno si era arrogato il diritto di nominare un presbitero "in cura d'anime", in quanto avrebbe assunto un beneficio ecclesiastico, e quindi, di amministrazione di ulteriori beni per la sua stessa sussistenza. Il Concilio chiese poi che l'arcidiacono fosse effettivamente ordinato diacono (can. 6) e condanna reati come la simonia e il concubinato (cann. $1 ; 7)^{87}$.

Si nota che l'arcidiacono non aveva ricevuto l'ordinazione. Questa prassi lascia intravedere che si trattasse di un mero incarico derivante dalla potestà di giurisdizione. D’altro canto, se l'arcidiacono aveva il compito di gestire beni, probabilmente il sorgere di tale abuso derivava dall'individuare che avesse capacità di gestione economica più che altre caratteristiche, neanche vocazionali.

Il Concilio Lateranense II, del $1139^{88}$, rinnova i canoni contro la simonia e il concubinato (cann. $1 ; 2 ; 7$ ), minaccia la decadenza della figura dellarcidiacono per chi rifiuta di farsi ordinare diacono (can. 10);

${ }^{84}$ Cf. K. Schatz, Storia dei concili. La Chiesa nei suoi punti focali, p. 98.

${ }^{85}$ Cf. R. Foreville, Lateran I-IV, Mainz 1970, pp. 39-50.

${ }^{86}$ Concilium Oecumenicum Lateranense I, Constitutio, can. 4, in: Conciliorum oecumenicorum decreta, ed. G. Alberigo, G. L. Dossetti, P. Joannou, C. Leonardi, P. Prodi, Bologna 1991, 166, 11-17.

${ }^{87}$ Cf. ibidem, 166, 1-5; 167, 1-5.

${ }^{88}$ Cf. K. Schatz, Storia dei concili. La Chiesa nei suoi punti focali, pp. 99-100. 
e aggiunge scomuniche per chi viola la "tregua di Dio" o fa di mestiere l'incendiario delle messi ${ }^{89}$.

Il Concilio Lateranense III, del $1179^{90}$, stabiliva che l'arcidiacono dovesse avere almeno 25 anni e che fosse veramente ordinato diacono, pena la decadenza dall'ufficio ${ }^{91}$. Si introdusse l'arcidiacono come visitatore delle chiese col vescovo (can. 4$)^{92}$. Il Concilio riprovava la prassi di vescovi e arcidiaconi di sospendere e scomunicare senza aver fatto procedere l'atto da un richiamo e da una ammonizione del reo (can. 6) ${ }^{93}$. La lettura di queste disposizioni fanno emergere la figura dellarcidiacono piuttosto che del diacono. Questo ufficio era presentato come centro di potere spesso slegato con lo stato diaconale, vista l'insistenza dei Concili nel richiedere lordinazione. Larcidiacono aveva grandi poteri amministrativi e fiscali, emetteva pene canoniche, aveva potere sulle parrocchie, tanto da visitarle e nominare i parroci, anche se la prassi era riprovata ${ }^{94}$.

Le cose non cambiano col passare del tempo; tanto che alla richiesta del Concilio di Trento della residenza nelle rispettive diocesi per i vescovi, questi la dicevano inutile fino a quando il loro potere era reso pressoché nullo, ristretto ed esautorato dalla ingerenza dei laici, dall'ingerenza della curia romana, dalla piaga delle esenzioni personali, dal potere dei capitoli, e dallo strapotere dell'arcidiacono ${ }^{95}$.

${ }^{89}$ Cf. Concilium Oecumenicum Lateranense II, Constitutio, in: Conciliorum oecumenicorum decreta, ed. G. Alberigo, G. L. Dossetti, P. Joannou, C. Leonardi, P. Prodi, Bologna 1991, 173, 1-11; 174, 6-16; 175, 11-14; 177, 15-29.

${ }^{90}$ Cf. K. Schatz, Storia dei concili. La Chiesa nei suoi punti focali, p. 101; A. Paravicini Bagliani, Il trono di Pietro. L'universalità del papato da Alessandro III a Bonifacio VIII, Roma 1996, pp. 65-71.

${ }^{91}$ Cf. Concilium Oecumenicum Lateranense III, Constitutio, in: Conciliorum oecumenicorum decreta, ed. G. Alberigo, G. L. Dossetti, P. Joannou, C. Leonardi, P. Prodi, Bologna 1991, 188, 19-28.

${ }^{92}$ Cf. ibidem, 189, 3-27.

${ }^{93}$ Cf. ibidem, 190, 8-33.

94 Cf. S. Zardoni, I diaconi nella chiesa. Ricerca storica e teologica sul diaconato, Bologna 1991², pp. 47-48; R. Selejdak, Diakonat stały w świetle Biblii i historii Kościoła, Częstochowa 2002, pp. 96; idem, Diakonat stały w świetle dokumentów Soboru Watykańskiego II, Posoborowego Urzędu Nauczycielskiego Kościoła i narodowych «Rationes institutionis diaconorum permanentium», Warszawa 2010, pp. 65-66.

${ }_{95}$ Cf. H. Jedin, Chiesa della fede. Chiesa della storia, Brescia 1972, pp. 275-315. 


\section{IL CONCILIO DI TRENTO}

Il Concilio di Trento ${ }^{96}$ si occupò del diaconato nella sessione XXIII del 15 luglio $1563^{97}$, sia nei canoni dottrinali sia in quelli de reformatione:

Il ministero annesso ad un sacerdozio così santo è cosa divina, fu perciò conveniente che, per esercitarlo più degnamente e con maggiore venerazione, nell'ordinata articolazione della Chiesa vi fossero più ordini di ministri e diversi fra loro, che servissero, per ufficio loro proprio, nel sacerdozio, e fossero così distribuiti, che quelli che fossero stati già insigniti della tonsura, attraverso gli ordini minori salissero ai maggiori. La Sacra Scrittura, infatti, nomina espressamente non solo i sacerdoti, ma anche i diaconi, ed insegna con parole solenni quello cui si deve sommamente badare nella loro ordinazione. E si sa che fin dall'inizio della Chiesa erano in uso i nomi degli ordini seguenti e i ministeri propri a ciascuno di essi: accolito, esorcista, lettore, ostiario, quantunque non con pari grado. Il suddiaconato, inoltre, dai padri e dai sacri concili è considerato tra gli ordini maggiori; e leggiamo in essi, frequentissimamente, anche quanto riguarda gli ordini minori ${ }^{98}$.

La preoccupazione del Concilio tridentino era dare rilevanza sacramentale all'Ordine. Secondo tale disposizione, il diaconato è menzionato esplicitamente dalla Scrittura, mentre gli ordini minori (suddiacono, accolito, esorcista, lettore, ostiario) sono ricordati fin dall'inizio della storia della Chiesa. Il suddiaconato era ritenuto dai Concili e dai padri un ordine maggiore. Lordine sacro era un vero e proprio sacramento istituto da Cristo (cap. 3; cann. 1; 3), che imprime il carattere che non rende più possibile un ritorno allo stato laicale, e che distingueva dal sacerdozio dei fedeli (cap. 4; can. 4). Relativamente all'ordine, la dottrina non era stata conclusa in quanto era fonte di discussione la sacramentalità dell'episcopato; non era chiaro se il carattere era di tutti gli "ordini", maggiori e minori, dato che tutti appartenevano allordine sacro 99.

${ }^{96}$ Cf. G. Martina, Storia della Chiesa. Da Lutero ai nostri giorni, Brescia 1997, pp. 241-262.

97 Cf. K. Schatz, Storia dei concili. La Chiesa nei suoi punti focali, p. 196.

98 Concilio di Trento, in: Enchiridion sul diaconato. Le fonti e i documenti ufficiali della Chiesa, a cura di E. Petrolino, Città del Vaticano 2009, pp. 125-126.

99 Cf. S. Zardoni, I diaconi nella chiesa. Ricerca storica e teologica sul diaconato, p. 48 . 
Il diaconato, così come gli altri ordini, era ritenuto un "gradino" che tendeva e conduceva verso il sacerdozio. Gli "ordini" fino al diaconato compreso non sembravano possedere una ragione intrinseca di esistenza per un proprio ministero, ma erano una sorta di progressiva preparazione e investitura in vista del sacerdozio.

Era fissata l'età per la ricezione: un minimo di 22 anni di età per il suddiaconato, 23 per il diaconato, 25 per il presbiterato (can. 12); per essere ordinati suddiaconi e diaconi, i candidati dovevano godere di buona reputazione, ed aver esercitato gli ordini minori. Quanto allesercizio del loro ministero che doveva essere di almeno un anno, il can. 13 si limitava a dire che:

servano alle chiese alle quali sono affidati, e sappiano che è sommamente opportuno che almeno nelle domeniche e nelle solennità, quando servono all'altare, facciano la sacra comunione ${ }^{100}$.

Inoltre il Concilio risponde ai protestanti che ritenevano inutili gli ordini sia minori che maggiori:

Perché le funzioni dei santi ordini, dal diaconato allostiariato, lodevolmente accolte nella Chiesa fin dai tempi degli apostoli, e in molti luoghi per lungo tempo interrotte, siano rimesse in uso secondo i sacri canoni, e non siano criticate dagli eretici come inutili, il santo sinodo, desiderando vivamente di rimettere in uso quellantica usanza. Stabilisce che, in futuro, tali ministeri non siano esercitati se non da quelli che sono costituiti in questi ordini. Il Concilio esorta, quindi, nel Signore, tutti e singoli i prelati e comanda loro di far in modo - per quanto è possibile - che nelle chiese cattedrali, collegiate e parrocchiali della loro diocesi, dove un popolo numeroso e i proventi della chiesa lo permettono, queste funzioni vengano ripristinate, assegnando a quelli che le esercitano uno stipendio sui redditi di qualche beneficio semplice o della fabbrica della chiesa, se vi fossero dei proventi, o dell'uno e dell'altra. Se poi questi chierici, fossero negligenti, siano multati di una parte degli emolumenti o addirittura privati di essi, a giudizio dellordinario. Se, inoltre, non si trovassero dei chierici celibatari per esercitare i quattro ordini minori, potranno essere loro sostituiti anche dagli sposati di onesta vita, adatti

100 Concilio di Trento, can. 13, 724, 36-725, 14. 
a questi uffici, purché non bigami e a condizione che in chiesa portino la tonsura e l'abito clericale ${ }^{101}$.

Il Concilio stabiliva che le funzioni degli ordini dovevano essere esercitate solo da chi era stato costituito nellordine stesso, contro l'abuso di esercitare senza essere stato costituito nel rispettivo ordine.

Si stabiliva di costituire in tali ordini chierici celibi. Nel caso nella comunità non fossero stati presenti, i chierici potevano essere sostituiti con uomini coniugati di vita specchiata, ma solo per accolitato, esorcistato, lettorato ed ostiariato; quindi sia per il diaconato come per il suddiaconato rimaneva ferma la legge del celibato.

In questa prospettiva, il Concilio chiedeva che i capitoli canonicali fossero composti per metà da presbiteri, e per l'altra metà da diaconi e suddiaconi ${ }^{102}$.

Il canone tridentino non parla esplicitamente di un diaconato permanente o comunque non potremmo assimilare il diaconato espresso da Trento a quello espresso nel Concilio Vaticano $\mathrm{II}^{103}$. Lo statuto personale del diacono che proviene dalla scarsa trattazione del Concilio tridentino, comunque afferma una rilevanza ecclesiale per il diacono.

Nell'ambito dello statuto personale si indica che esso appartiene all'Ordine e che l'Ordine è un Sacramento. Si aggiunge che esso costituisce un "gradino" verso il sacerdozio. Questo chiaramente risponde allopposizione protestante che sconfessava la sacramentalità dell'Ordine. Nello statuto funzionale non si aggiunge nulla in più rispetto alle sue mansioni liturgiche eccetto il dato di dover essere stati costituiti nell'Ordine per poterlo esercitare. In generale il Concilio di Trento non risulta essere innovatore rispetto alla disciplina precedente.

Larricchimento potrebbe ravvisarsi nella possibilità data agli uomini sposati e di vita specchiata di "sostituire" i chierici negli ordini minori, ma non nel diaconato e suddiaconato che restano appannaggio

101 Ibidem, can. 17, p. 126.

102 Cf. Concilio di Trento, can. 12 de Reformatione, in: Conciliorum oecumenicorum decreta, ed. G. Alberigo, G. L. Dossetti, P. Joannou, C. Leonardi, P. Prodi, Bologna 1991, 742, 27-34.

${ }^{103}$ Cf. S. Zardoni, I diaconi nella chiesa. Ricerca storica e teologica sul diaconato, pp. $48-50$. 
dei chierici, ovvero di coloro che avendo ricevuto la tonsura erano tenuti al celibato.

\section{CONCLUSIONE}

Lo studio presente rileva che i Concili della Chiesa svoltisi sia in Occidente che in Oriente dal IV al XVI secolo hanno emanato diverse disposizioni riguardanti l'identità teologica e giuridica dei diaconi nonché il loro statuto personale e funzionale nelle comunità cristiane. I canoni conciliari in modo chiaro definivano le prerogative sacramentali dei diaconi. Stabilivano, tra l'altro, che in mancanza del vescovo, o se in sua presenza fosse stato delegato, solo il presbitero poteva cresimare, e non il diacono. Relativamente all'Eucaristia, nei Concili antichi sovente si trovano ingiunzioni disciplinari che proibiscono ai diaconi di celebrare l'Eucaristia o che diffidano da altri tipi di abuso. I Concili insistevano continuamente, soprattutto nella Chiesa in Occidente, sullobbligo del celibato per i diaconi, e se uxorati, sulla vita in castità e continenza. Largomentazione che veniva citata in tali casi era: i diaconi devono osservare la castità domandata da Dio. La loro purezza, compresa in modo integrale, cioè del corpo e dello spirito, era indicata come una condizione della efficacia della loro preghiera e del servizio ecclesiale. In caso di mancato rispetto della norma i canoni conciliari richiedevano loro espulsione dall'ufficio. Le disposizioni dei Concili non permettevano ai diaconi di dedicarsi a occupazioni che potevano esporre al danno la sua morale, ed inoltre, di non impegnarsi nelle occupazioni che rendevano impossibile oppure ostacolavano l'esecuzione dei doveri legati con l'incarico ecclesiale. Vietati erano anzitutto le occupazioni che domandavano i frequenti viaggi come anche i mestieri che impegnavano tutto il tempo. I diaconi non dovevano impegnarsi negli affari di carattere economico. Generalmente i Concili biasimavano i mestieri legati direttamente con il denaro perché sempre creavano il sospetto d'agire per motivo di cupidigia. Le disposizioni conciliari riguardavano anche il vestiario e il modo di presentarsi. Raccomandavano una certa moderazione, mettendo in rilievo che non tutto conveniva ai diaconi. Il modo di vestirsi e il comportamento, senza sfoggio e senza guardare la moda, doveva rispettare la spiritualità degli 
uomini dedicati al servizio divino. Infine, i Concili stabilivano le normative concernenti l'attività caritativa dei diaconi per evitare i conflitti tra loro ed i vescovi, ordini religiosi e mendicanti, e laici.

\section{BIBLIOGRAFIA}

Agrimi J., Crisciani C., Malato, medico e medicina nel medioevo, Torino 1980.

Andenna G., Pievi e parrocchie in Italia centro-settentrionale, in: Pensiero e sperimentazioni istituzionali nella Societas Christiana, 1046-1250. Atti della sedicesima Settimana internazionale di studio, Mendola, 26-31 agosto 2004, a cura di G. Andenna, Milano 2007, pp. 364-373.

Badot Ph., De Deckers D., Historicité et actualité des canones disciplinaires du concile d'Elvire, "Augustinianum" 37 (1997), p. 311-325.

Barbieri R., Uomini e tempo medievale, Milano 1988.

Bartoli M., Block W., Mastromatteo A., Storia della spiritualità francescana. I - Secoli XIII-XVI, Bologna 2017.

Baus K., Storia della Chiesa tra oriente e occidente, Milano 1992.

Bienert W.A., Das vornicänische homoousios als Ausdruck der Rechtgläbigkeit, "Zeitschrift für Kirchengeschichte" 90 (1979), pp. 5-29.

Bucci O., Piatti P., Storia dei concili ecumenici, Roma 2014.

Capitularia I, in: Monumenta Germaniae historica, I, a cura di A. Boretius, Hannoverae 1883 , p. 239.

Castellucci E., Il ministero ordinato, Brescia 2002.

Chidenius J., Medieval institutions and the Old Testament, Helsinki Helsingfors 1965.

Chittolini G., Miccoli G., La Chiesa e il potere politico dal Medioevo alletà contemporanea, Torino 1986.

Concilio di Agde, in: Enchiridion fontium historicae Ecclesiasticae Antiquae, ed. C. Kirch, Barcellona 1973, n. 963-966.

Concilio di Adge, in: Concilia Galliae a. 314-506, can. 9, ed. C. Munier, Corpus Christianorum Series Latina (CCSL 148), Turnhout 2001, p. 197.

Concilio di Ancira, in: Enchiridion fontium historicae Ecclesiasticae Antiquae, ed. C. Kirch, Barcellona 1973, n. 381.

Concilio Arles, in: Concilia Galliae a. 314-506, can. 43, ed. C. Munier, Corpus Christanorum Series Latina (CCSL 148), Turnhout 2001, p. 122.

Concilio di Arles I, in: Enchiridion fontium historicae Ecclesiasticae Antiquae, ed. C. Kirch, Barcellona 1973, n. 373.

Concilio di Cartagine, in: Concilia Africae a. 345-525, cann. 11; 12; 13, ed. C. Munier, Corpus Christanorum Series Latina (CCSL 149), Turnhout 1974, p. 37.

Concilio di Nicea, Canones, in: Conciliorum oecumenicorum decreta, ed. J. Alberigo, P. Joannou, C. Leonardi, P. Prodi, Bologna 1991, nn. 13, 30-38; 14, 1-8. 
Concilio di Orange, in: Enchiridion fontium historicae Ecclesiasticae Antiquae, ed. C. Kirch, Barcellona 1973, nn. 871-872.

Concilio di Orléans, Concilia Galliae a. 511-695, can. 8, ed. C. De Clercq, Corpus Christianorum Series Latina (CCSL 148 A), Turnhout 1963, p. 8.

Concilio Quinisesto, in: Enchiridion fontium historicae Ecclesiasticae Antiquae, ed. C. Kirch, Barcellona 1973, n. 1093.

Concilio di Toledo, in: Enchiridion fontium historicae Ecclesiasticae Antiquae, ed. C. Kirch, Barcellona 1973, nn. 707; 974.

Concilio di Tours, in: Concilia Galliae a. 314-506, can. 3, ed. C. Munier, Corpus Christanorum Series Latina (CCSL 148), Turnhout 2001, p. 143.

Concilio di Trento, in: Conciliorum oecumenicorum decreta, can. 12 de Reformatione, ed. G. Alberigo, G. L. Dossetti, P. Joannou, C. Leonardi, P. Prodi, Bologna 1991, 742, 27-34.

Concilio di Trento, in: Enchiridion sul diaconato. Le fonti e $i$ documenti ufficiali della Chiesa, a cura di E. Petrolino, Città del Vaticano 2009, pp. 125-126.

Concilio di Vaison, in: Concilia Galliae a. 511-695, can. 2, ed. C. De Clercq, Corpus Christianorum Series Latina (CCSL 148 A), Turnhout 1963, p. 79.

Concilio di Vannes, Concilia Galliae a. 314-506, can. 11, ed. C. Munier, Corpus Christanorum Series Latina (CCSL 148), p. 154.

Concilium Oecumenicum Lateranense I, Constitutio, can. 4, in: Conciliorum oecumenicorum decreta, ed. G. Alberigo, G. L. Dossetti, P. Joannou, C. Leonardi, P. Prodi, Bologna 1991, 166, 11-17.

Concilium Oecumenicum Lateranense II, Constitutio, in: Conciliorum oecumenicorum decreta, ed. G. Alberigo, G. L. Dossetti, P. Joannou, C. Leonardi, P. Prodi, Bologna $1991,173,1-11 ; 174,6-16 ; 175,11-14 ; 177,15-29$.

Concilium Oecumenicum Lateranense III, Constitutio, in: Conciliorum oecumenicorum decreta, ed. G. Alberigo, G. L. Dossetti, P. Joannou, C. Leonardi, P. Prodi, Bologna 1991, 188, 19-28.

Cruzel E., L'organizzazione ecclesiastica nelle campagne, "Reti medievali" 1 (2010), pp. 417-435.

Demurger A., Chevaliers du Christ. Les Ordres religieux-militaires au Moyen Áge (XI e-XVI e siècle), Paris 2002.

Eginardo, Vita Karoli Magni, cap. 3, in: Sources d'histoire médiévale. IX-XIV siècle, a cura di G. Brunel, E. Lalou, Paris 1992, p. 23.

Février P.A., Césaire et la Gaule méridionale au VI siècle, in: Césaire d’Arles e la Christianisation de la Provence. Actes des journées «Césaire» (Aix-en-Provence-Arles-Lérins, 3-5 novembre 1988, 22 avril 1989), a cura di D. Bertrand, Paris 1994, pp. $45-73$.

Foreville R., Lateran I-IV, Mainz 1970.

Gandino G., Contemplare l'ordine: intellettuali e potenti dellalto Medioevo, Napoli 2004.

Gessel W., Nizäa 325: Die Doppelstruktur eines Reichskonzils, in: Synodale Strukturen der Kirche, Donauwörth 1977, pp. 61-77. 
Gregorius PP. I., Lettere VI, 4, ed. J. P. Migne, Patrologiae Cursus Completus. Series Latina (PL, 77), Parisiis 1862, pp. 796-840.

Hammann G., Storia del diaconato, Magnano 2004.

Hertling I., Bulla A., Storia della Chiesa. La penetrazione dello spazio umano ad opera del cristianesimo, Roma 2001.

Jedin H., Chiesa della fede. Chiesa della storia, Brescia 1972.

Kasper W., Nocchiero in mezzo alla tempesta. Il ministero episcopale secondo Tommaso d'Aquino, in: Teologia e Chiesa, 2, a cura di W. Kasper, Brescia 2001, pp. 109-135.

Le Goff J., San Francesco d'Assisi, Roma-Bari 2000.

Martina G., Storia della Chiesa. Da Lutero ai nostri giorni, Brescia 1997.

Massagrande F., Il Sacramento dell'Ordine: il presbiterato nella «Summa Theologiae» di S. Tommaso d'Aquino, Esperienza e Teologia, 24 (2008), pp. 97-102.

Maurer W., Die christliche Diakonie im Mittelalter, in: Das diakonische Amt der Kirche, a cura di H. Krimm, Stuttgart 1965, p. 130-142.

Merlo G.G., Esperienze religiose e opere assistenziali nei secoli XII e XIII, Torino 1987.

Morichetti M., La predicazione di Cesario di Arles al superstizioso, in: Evangelizzazione dell'Occidente dal terzo all'ottavo secolo, a cura di L. Bacci, I. Mazzini, Roma 2001, pp. 105-123.

Paravicini Bagliani A., Il trono di Pietro. L’universalità del papato da Alessandro III a Bonifacio VIII, Roma 1996.

Penone D., I domenicani nei secoli. Panorama storico dell'Ordine dei frati predicatori, Bologna 1998.

Piana M., C. Carlsson C., Archaeology and Architecture of the Military Orders: New Studies, London 2016.

Pirenne H., Les villes du Moyen Áge, essaì d'histoire économique et sociale, Paris 1927.

Pitz E., Europäisches Städtewesenund Bürgertum von der Spätantike bis zum holen Mittelalten, Darmastadt 1991.

Rigali J., Découvrir les ministèrs, Paris 2001.

Schatz K., Storia dei concili. La Chiesa nei suoi punti focali, Bologna 1999.

Scheinder R., Karl der Grosse, politisches Sendungsbewusstein und mission, in: Kirchengeschichte als Missiongeschichte, II, a cura di H. Frohnes, H. W. Gensichen, G. Kretschmar, München 1978, pp. 227-248.

Selejdak R., Diakonat stały w świetle Biblii i historii Kościoła, Częstochowa 2002.

Selejdak R., Diakonat stały w świetle dokumentów Soboru Watykańskiego II, Posoborowego Urzędu Nauczycielskiego Kościoła i narodowych «Rationes institutionis diaconorum permanentium», Warszawa 2010.

Sinodo di Elvira, in: Enchiridion fontium historicae Ecclesiasticae Antiquae, a ed. C. Kirch, Barcellona 1973, nn. 336, 339, 401.

Siricio, Littera directa ad decessorem ad Himerium tarraconensem episcopum, 10 febbraio 285, in: Enchiridion symbolorum, ed. H. Denzinger, n. 185, pp. 103-104.

Sottomayor Muro M., Ubiña J.F., El Concilio de Elvira y su tiempo, Granada 2005. 
Statuta Ecclesiae Antiqua, in: Concilia Galliae a. 314-506, can. 27, ed. C. Munier, Corpus Christanorum Series Latina (CCSL 148), Turnhout 1974, p. 171.

Thomae Aquinatis, Summa Theologiae, wyd. BACr, vol. 4, pars III, Madrid 1958.

Zardoni S., I diaconi nella Chiesa. Ricerca storica e teologica sul diaconato, Bologna $1991^{2}$. 